\title{
No first ionization potential fractionation in the active stars AR Piscium and AY Ceti ${ }^{\star}$
}

\author{
J. Sanz-Forcada ${ }^{1}$, L. Affer ${ }^{2}$, and G. Micela ${ }^{2}$ \\ ${ }^{1}$ Laboratorio de Astrofísica Estelar y Exoplanetas, Centro de Astrobiología / CSIC-INTA, LAEFF Campus, PO Box 78, \\ 28691 Villanueva de la Cañada, Madrid, Spain \\ e-mail: jsanz@laeff.inta.es \\ 2 INAF - Osservatorio Astronomico di Palermo G. S. Vaiana, Piazza del Parlamento, 1, 90134 Palermo, Italy \\ e-mail: [affer;giusi]@astropa.inaf.it
}

Received 13 March 2009 / Accepted 1 July 2009

ABSTRACT

\begin{abstract}
Context. The comparison of coronal and photospheric abundances in cool stars is an essential question to resolve. In the Sun an enhancement of the elements with low first ionization potential (FIP) is observed in the corona with respect to the photosphere. Stars with high levels of activity seem to show a depletion of elements with low FIP when compared to solar standard values; however, the few cases of active stars in which photospheric values are available for comparison lead to confusing results, and an enlargement of the sample is mandatory for solving this longstanding problem.

Aims. We calculate in this paper the photospheric and coronal abundances of two well known active binary systems, AR Psc and AY Cet, to get further insight into the complications of coronal abundances.

Methods. Coronal abundances of 9 elements were calculated by means of the reconstruction of a detailed emission measure distribution, using a line-based method that considers the lines from different elements separately. Photospheric abundances of 8 elements were calculated using high-resolution optical spectra of the stars.

Results. The results once again show a lack of any FIP-related effect in the coronal abundances of the stars. The presence of metal abundance depletion (MAD) or inverse FIP effects in some stars could stem from a mistaken comparison to solar photospheric values or from a deficient calculation of photospheric abundances in fast-rotating stars.

Conclusions. The lack of FIP fractionation seems to confirm that Alfvén waves combined with pondermotive forces are dominant in the corona of active stars.
\end{abstract}

Key words. stars: coronae - stars: abundances - stars: individual: AR Psc - X-rays: stars - line: identification stars: individual: AY Cet

\section{Introduction}

One of the most debated issues in stellar astrophysics is whether the coronal abundances are similar to the photospheric counterparts in cool stars. A different composition in the corona and the photosphere would indicate a physical process taking place somewhere between the cooler photospheric material and the hotter corona. Such a process should be capable of distinguishing between different elements based on a certain physical variable. A pattern related to that variable would help understanding the physical processes taking place in the coronal loops. Such a pattern has been observed in the Sun, which shows, on average, an enhancement of elements with a low first ionization potential (FIP) in the corona with respect to the photosphere. The so called "FIP effect" is actually observed in the solar corona and slow wind, but is absent in coronal holes or fast wind (Laming et al. 1995; Feldman \& Laming 2000). Stars similar to the Sun, such as $\alpha$ Cen, show a similar FIP effect (Drake et al. 1997; Raassen et al. 2003). Less active stars, such as Procyon (F4IV), do not show any fractionation (Raassen et al. 2002; Sanz-Forcada et al. 2004). Intermediate-activity stars, like $\epsilon$ Eri, 36 Oph or 70 Oph present a much lower FIP effect, if any is present (Laming et al. 1996; Sanz-Forcada et al. 2004; Wood \& Linsky 2006; Ness \& Jordan 2008).

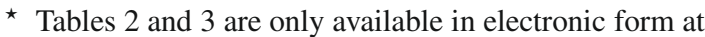
http://www . aanda.org
For the most active stars, the results are more uncertain. Their coronal composition is relatively easy to determine once high spectral resolution is available. But their high rotation rates hamper the measurements of photospheric composition due to line broadening, making the comparison more difficult. The photospheric composition can only be well calculated for stars with low projected rotational velocity $(v \sin i)$. Initial studies of active stars made with XMM-Newton and Chandra high-resolution spectra found a very different pattern from the Sun (e.g. Güdel 2004, and references therein). The elements with low FIP would actually be underabundant in the corona, a "metal abundance depletion" ("MAD syndrome", Schmitt et al. 1996), or alternatively, the elements with high FIP would be enhanced in the corona (the "inverse FIP effect", Brinkman et al. 2001). A caveat of many of the active stars is that their coronal abundances are actually compared to the solar photosphere, which at least yields to risky conclusions (Favata \& Micela 2003). Moreover, most active stars with photospheric abundances calculated have large $v \sin i$, with broad photospheric lines. As a result, their abundances determination could actually carry some hidden errors not assessed well by formal calculations. This is the case for II Peg, AB Dor, or AR Lac (see Table 1).

Theoretical explanations of the FIP effect are found in Laming (2004). The authors also propose that Alfvén waves, combined with pondermotive forces, would explain the observed FIP effect on the Sun, pointing towards the disappearance of any 
Table 1. Fractionation effects in other stars with known photospheric and coronal abundances.

\begin{tabular}{|c|c|c|c|c|c|c|c|}
\hline Star & HD & Sp. Type & $\begin{array}{c}L_{\mathrm{X}} \\
\left(\mathrm{erg} \mathrm{s}^{-1}\right)\end{array}$ & $L_{\mathrm{X}} / L_{\mathrm{bol}}$ & $\begin{array}{c}v \sin i \\
\mathrm{~km} \mathrm{~s}^{-1}\end{array}$ & FIP effect? & Reference $^{a}$ \\
\hline Sun & & $\mathrm{G} 2 \mathrm{~V}$ & $\begin{array}{l}27.5 \\
\end{array}$ & -6.1 & $\ldots$ & FIP effect & FE00 \\
\hline Procyon & 61421 & F4IV & 27.9 & -6.5 & 6.1 & No FIP effect & SF04, RA02 \\
\hline$\epsilon$ Eri & 22049 & $\mathrm{~K} 2 \mathrm{~V}$ & 28.2 & -5.1 & 2.4 & Small/No FIP effect & SF04 \\
\hline$\xi \mathrm{UMa} \mathrm{B}$ & 98230 & $\mathrm{G} 5 \mathrm{~V} /[\mathrm{KV}]$ & 29.5 & -4.3 & 2.8 & (No FIP effect) & BA05 \\
\hline$\lambda$ And & 222107 & G8III/? & 30.4 & -4.5 & 7.3 & No FIP effect & SF04 \\
\hline Capella & 34029 & G1III/G8III & 30.5 & -5.3 & 32.7 & (No FIP effect) & BR00 \\
\hline II Peg & 224085 & $\mathrm{~K} 2 \mathrm{IV} / \mathrm{M} 0-3 \mathrm{~V}$ & 31.1 & -2.8 & 21 & Small inverse FIP & HU01 \\
\hline AB Dor & 25647 & K1IV & 30.1 & -3.0 & 90 & Inverse FIP & SF03 \\
\hline AR Lac & 210334 & G2IV/K0IV & 30.9 & -3.4 & $46 / 81$ & Small inverse FIP & HU03 \\
\hline V851 Cen & 119285 & K2IV-III/? & 30.8 & -3.5 & 6.5 & No FIP effect & SF04 \\
\hline AR Psc & 8357 & G7V/K1IV & 30.7 & -3.3 & 7.0 & No FIP effect & This work \\
\hline AY Cet & 7672 & WD/G5III & 31.0 & -4.2 & 4.6 & No FIP effect & This work \\
\hline
\end{tabular}

Note: $L_{\mathrm{X}}\left(\mathrm{erg} \mathrm{s}^{-1}\right)$ calculated in the range 5-100 $\AA(0.12-2.4 \mathrm{keV})$.

${ }^{a}$ References for FIP effect: BA05 (Ball et al. 2005), BR00 (Brickhouse et al. 2000), FE00 (Feldman \& Laming 2000), HU01 (Huenemoerder et al. 2001), HU03 (Huenemoerder et al. 2003), RA02 (Raassen et al. 2002), SF04 (Sanz-Forcada et al. 2004), SF03 (Sanz-Forcada et al. 2003b).

fractionation of stars with lower or higher activity levels. The same mechanism could also explain the inverse FIP effect, if such exists, by reflecting the Alfvén waves in the chromosphere. Alfén waves are also being suggested in recent years as responsible for the energy transportation between the outer convective layers of the star and the much hotter corona (e.g. Erdélyi \& Fedun 2007; De Pontieu et al. 2007, and references therein), one of the most important problems unresolved in stellar astrophysics. Both questions, the energy transportation and the FIP fractionation, could actually be connected.

It is thus essential to understand whether active stars suffer an inverse FIP effect or just no significant fractionation with respect to the photospheric composition. Given the problems measuring the photospheric abundances, we should mainly trust those results for stars with low $v \sin i$. Sanz-Forcada et al. (2004) showed the case of two active stars, $\lambda$ And and V851 Cen, for which coronal and photospheric abundances are consistent, and no effect related to FIP would be present. Stars such as Capella (which shows solar photospheric abundances, Brickhouse et al. 2000; Argiroffi et al. 2003; Audard et al. 2003) show no inverse FIP effect either, although their photospheric abundance is poorly known $([\mathrm{Fe} / \mathrm{H}]=-0.16, \mathrm{McWilliam} \mathrm{1990)})$. Other cases of active stars with no sign of an inverse FIP effect, which are compared to poorly known photospheric iron abundances, are $\sigma^{2} \mathrm{CrB}$ (Suh et al. 2005), EK Dra (Telleschi et al. 2005), and YY Men (Audard et al. 2004) ${ }^{1}$. The present situation is still confusing given the few cases described in the literature with both photospheric and coronal abundances well-calculated. In this work we present the results for two more RS CVn systems known to have narrow photospheric lines despite their high rotation and activity level.

Following Sanz-Forcada et al. (2004) we have chosen two active stars that are observed with their pole on, and therefore have low $v \sin i$. Thus, their optical spectra display narrow lines, allowing us to measure the photospheric abundances better. The two RS CVn binaries are well known X-ray

\footnotetext{
1 Although the authors claim that coronal iron is depleted in YY Men, the values in the corona and photosphere of the star are actually consistent, once reasonable uncertainties of 0.2 dex are considered for the photospheric iron, calculated from a low-resolution spectrum by Randich et al. (1993).
}

emitters. Their emission is attributed to coronal activity, powered by the fast rotation forced by the close binariety. AR Psc (G7V/K1IV, $v \sin i=7 \mathrm{~km} \mathrm{~s}^{-1}$, Nordström et al. 2004) and AY Cet (WD/G5III, $v \sin i=4.6 \mathrm{~km} \mathrm{~s}^{-1}$, Massarotti et al. 2008) were observed with the Extreme Ultraviolet Explorer Observatory (EUVE), giving a first glimpse of its coronal emission (SanzForcada et al. 2003a). Optical spectra also indicate a high level of chromospheric activity (Montes et al. 1997). Their EMD is similar to other active stars, either showing an inverse FIP effect, like AB Dor (Sanz-Forcada et al. 2003b; García-Alvarez et al. 2005, 2008), or no FIP-related fractionation, such as $\lambda$ And (Sanz-Forcada et al. 2004). In the case of AY Cet, it is not expected that its white dwarf companion contributes substantially to the X-rays band. Photospheric abundances were calculated by Ottmann et al. (1998) for AY Cet in only three elements: $[\mathrm{Fe} / \mathrm{H}]=-0.32,[\mathrm{Mg} / \mathrm{H}]=-0.22$, and $[\mathrm{Si} / \mathrm{H}]=-0.32$, with Asplund et al. (2005) values as reference. Shan et al. (2006) finds a low iron abundance in AR Psc, but no information is provided on the reference system used in the calculations.

The paper is divided as follows: in Sect. 2 the observations are described. Results are given in Sect. 3, with discussion in Sect. 4 , to finish with the conclusions.

\section{Observations}

Time was awarded (P.I. J. Sanz-Forcada) for observing highresolution X-rays spectra of AY Cet and AR Psc. The Chandra Low Energy Transmission Grating Spectrograph (LETGS) ( $\lambda \lambda \sim 3-175, \lambda / \Delta \lambda \sim 60-1000$, Weisskopf et al. 2002) observed AY Cet in May 2005 in combination with the High Resolution Camera (HRC-S). Data were reduced using the CIAO v4.0 package. The positive and negative orders were summed for the flux measurements. Lines formed in the first dispersion order, but contaminated with contribution from higher dispersion orders, were not employed in the analysis. Light curves were obtained from the LETG spectra (first and higher orders) of AY Cet with the background properly subtracted (Fig. 1). XMM-Newton observed AR Psc on 11 January 2006. XMM-Newton observes simultaneously with the RGS (Reflection Grating Spectrometer, den Herder et al. 2001) $(\lambda \lambda \sim 6-38 \AA, \lambda / \Delta \lambda \sim 100-500)$ and the EPIC (European Imaging Photon Camera) PN and MOS 

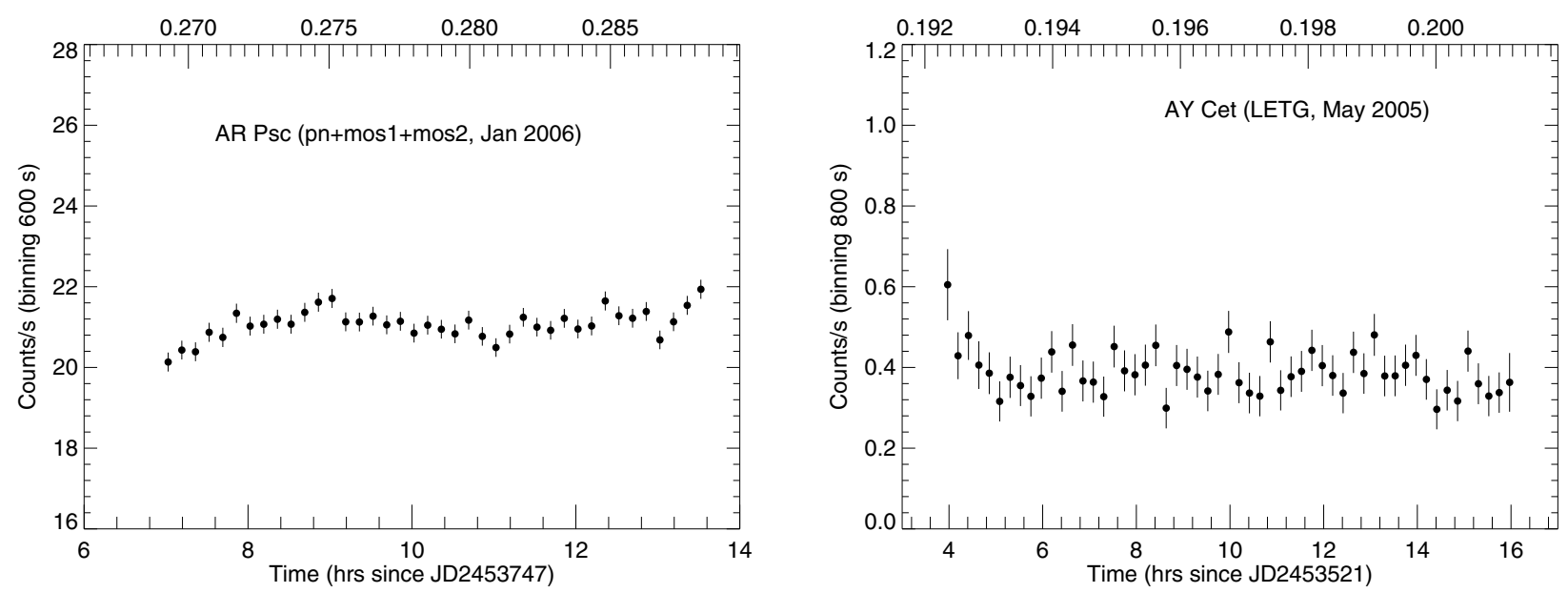

Fig. 1. Light curves of AR Psc (using all EPIC detectors) and AY Cet (using order +1 and -1 of LETGS) in X-rays. Upper axis indicate the orbital phase, assuming that secondary star is located behind the primary star at phase 0 .

detectors (sensitivity range $0.15-15 \mathrm{keV}$ and $0.2-10 \mathrm{keV}$, respectively). The RGS data were reduced using the standard SAS (Science Analysis Software) version 8.0.1 package, and the RGS 1 and 2 spectra were combined to improve the measurement of the line fluxes (Fig. 2). The EPIC light curve (Fig. 1) was constructed by combining the PN and MOS count rates in a circle around the target, with background regions properly subtracted using the SAS task epiclccorr, which also corrects from several instrumental effects.

Optical spectra (Fig. 3) were acquired on 27 and 28 November 2002 to calculate the photospheric abundances of the two stars, as part of a wider observational campaign. The detailed instrumental setup and observations are described well in Affer et al. (2005), so only a short description is given here. We used the high-resolution cross-dispersed echelle spectrograph SOFIN, mounted on the Cassegrain focus of the $2.56 \mathrm{~m}$ Nordic Optical Telescope (NOT) located at the Observatorio del Roque de Los Muchachos (La Palma, Spain). Exposure times ranged from 4 to $20 \mathrm{~min}$, resulting in high $S / N$ per pixel $(\approx 0.025 \AA / \mathrm{px})$ averaging at about 280 . A spectrum of a Th-Ar lamp was obtained following each stellar spectrum, ensuring accurate wavelength calibration. The total spectral range is 3900-9900 $\AA$, and the resolving power is $R=\lambda / \Delta \lambda \approx 80000$. The spectra were reduced with the standard software available within the CCDRED and ECHELLE packages of IRAF ${ }^{2}$. The analysis includes overscan subtraction, flat-fielding, removal of scattered light, extraction of one-dimensional spectra, wavelength calibration, and continuum normalization (see Affer et al. 2005, for further details). The $E W \mathrm{~s}$ were measured using the SPLOT task in IRAF, assuming a Gaussian profile for weak or moderately strong lines ( $E W \lesssim 100 \mathrm{~mA}$ ) and a Voigt profile for stronger lines. The accuracy (absolute error) is harder to assess. It almost certainly contains a systematic error due to the continuum location, because of the presence of interference fringes (which could not be completely removed) in the redder part of the stellar spectra, which cause a modulation of the local continuum. This error could be particularly important for the weak lines.

${ }^{2}$ IRAF (Image Reduction and Analysis Facility) is distributed by National Optical Astronomy Observatories, operated by the Association of Universities for Research in Astronomy, Inc., under cooperative agreement with the National Science Foundation.

\section{Results}

Light curves of AR Psc and AY Cet (Fig. 1) show no flares in these observations, although variability up to a $\sim 10 \%$ takes place in AR Psc. The small portion of the orbital period covered prevents us from identifying variability related to orbital phase in both stars. Both systems behave like the EUVE observations reported by Sanz-Forcada et al. (2003a). The spectra recorded by EUVE were of limited use because of low statistics. The large number of lines observed with XMM-Newton and Chandra have allowed us to construct a more accurate emission measure distribution (EMD) as a function of temperature, defined as $E M(T)=$ $\int_{\Delta T} N_{\mathrm{H}} N_{\mathrm{e}} \mathrm{d} V\left[\mathrm{~cm}^{-3}\right]$. We used a line-based analysis described in Sanz-Forcada et al. (2003b) and references therein. In short, individual line fluxes are measured ${ }^{3}$ (Tables 2,3) and then compared to a trial EMD, which is combined with the atomic emission model Astrophysical Plasma Emission Database (APED v1.3.1, Smith et al. 2001) in order to produce theoretical fluxes of the lines. The comparison of measured and modeled line fluxes result in an improved EMD that is used again to produce new modeled line fluxes. The iterative process result in a solution that is not unique, but reliably approximates the observed and modeled fluxes, and it presumably resembles the real EMD of the corona. Error bars were calculated using a Monte Carlo method that seeks the best solution for different line fluxes within their $1-\sigma$ errors (see Sanz-Forcada et al. 2003b, for more details). In our case the lines measured are formed at different temperatures and correspond to several elements. We took the caution to construct an initial EMD using only Fe lines and then progressively added lines of each element with overlapping temperatures to the analysis. The determined EMDs are displayed in Fig. 4 and Table 4. Coronal abundances (Fig. 5, Table 5) were calculated with the same process and then compared to the values measured in their own photospheres (see below). We used the solar photospheric values (Asplund et al. 2005) as reference. Some solar abundance values have changed between Anders \& Grevesse (1989) (used in Sanz-Forcada et al. 2004) and Asplund et al. (2005). Most notably, $[\mathrm{Fe} / \mathrm{H}]$ is now 7.45 instead of 7.67.

The photospheric abundances (Fig. 6, Table 5) were calculated through the analysis of the optical spectra, in which we

\footnotetext{
3 We then measured fluxes for interstellar medium absorption (ISM), using $\log N_{\mathrm{H}}=18.8$ (AY Cet) and $\log N_{\mathrm{H}}=18.3$ (AR Psc), although it is only important in a few lines.
} 
AR Psc XMM/RGS spectrum (25 ks)

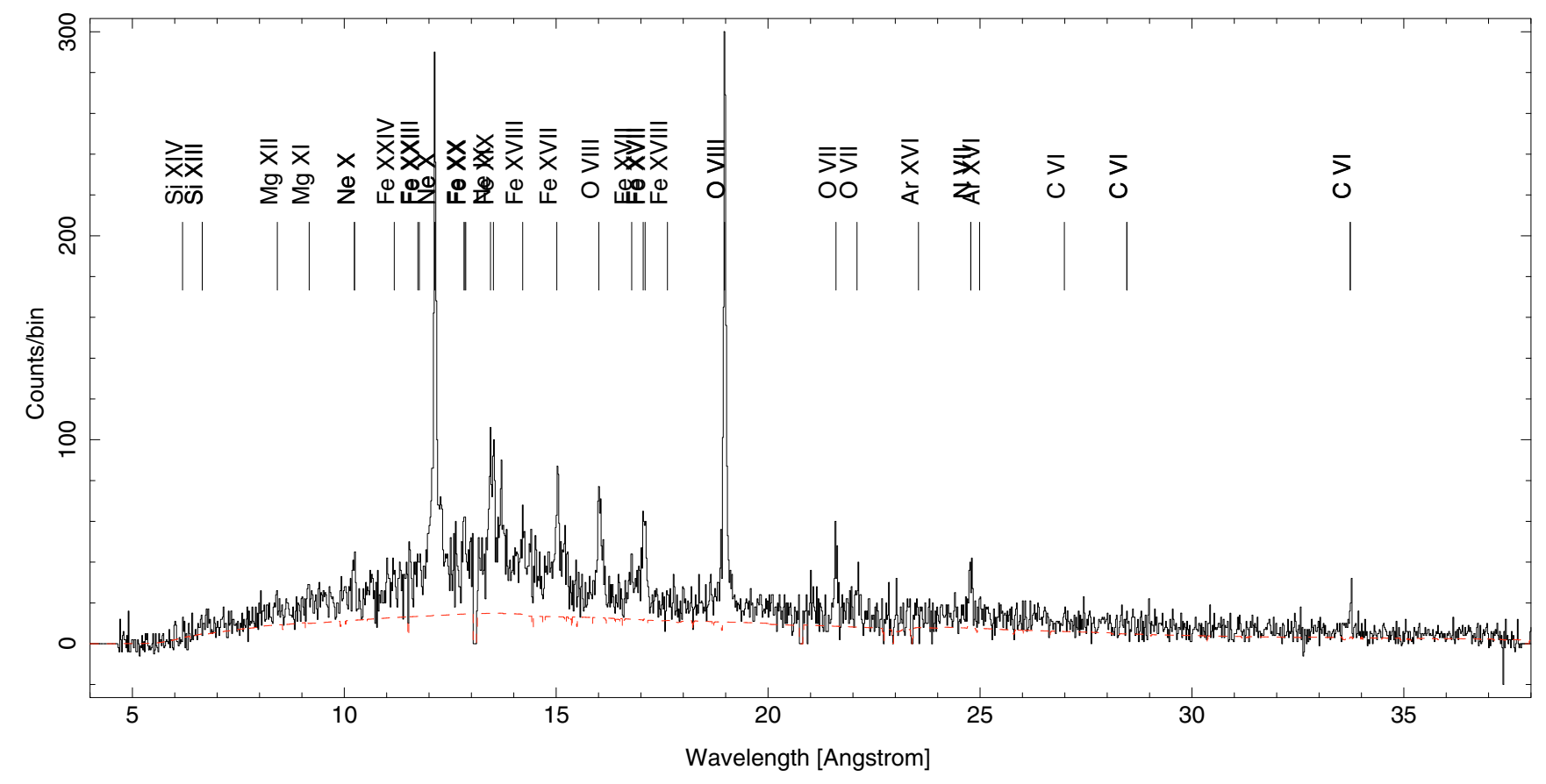

AY Cet Chandra/LETGS spectrum (43 ks)

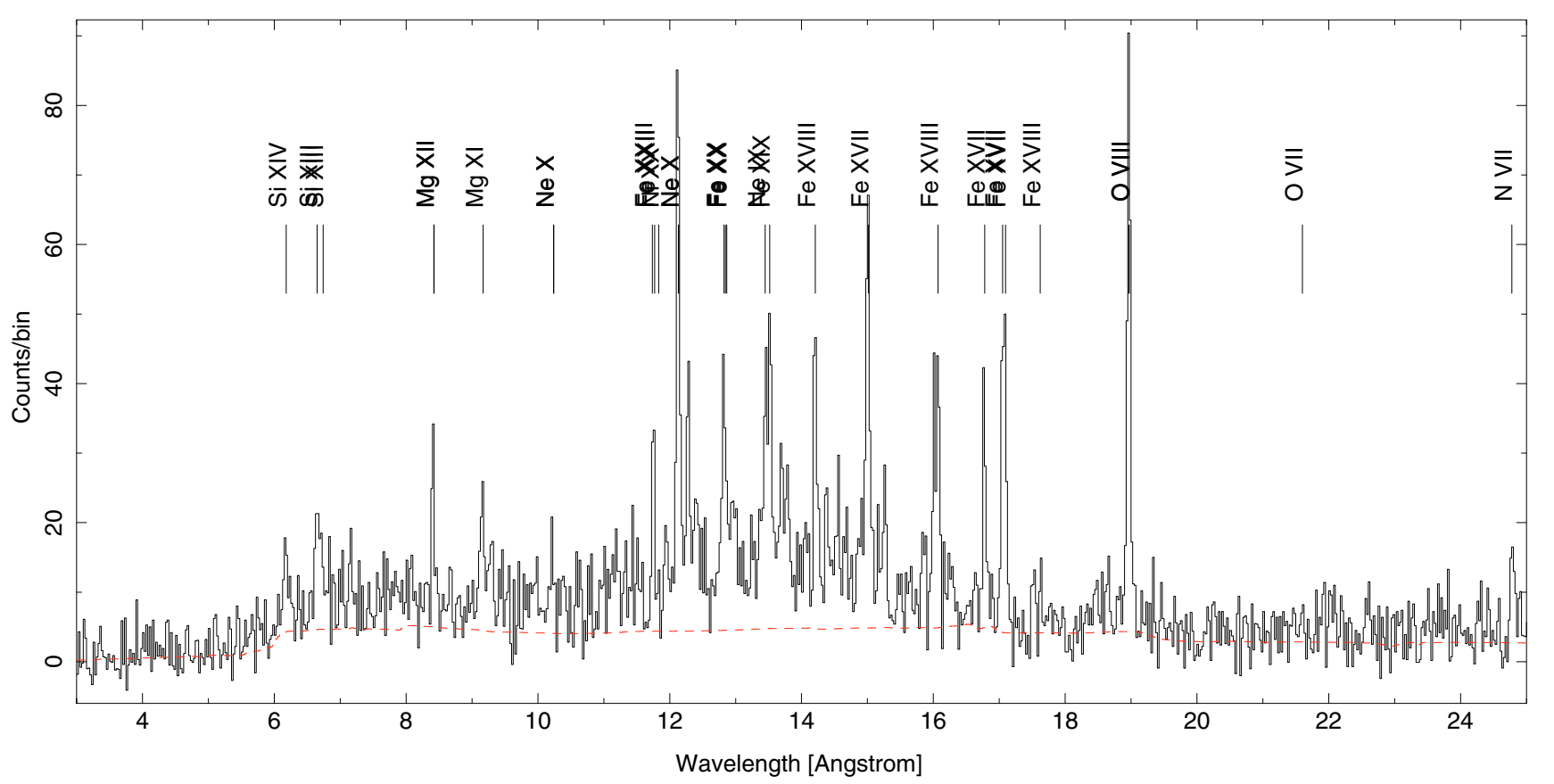

Fig. 2. AR Psc and AY Cet X-ray spectra. The dashed line represents the continuum predicted by the EMD.

select a group of lines that are free of blends, as explained in Affer et al. (2005) and Morel et al. (2003). To avoid the difficulty in defining the continuum in the blue part of the spectra, only lines with $\lambda>5500 \AA$ were selected. Lines that appeared asymmetric or showed an unusually large width were assumed to be blended with unidentified lines so were discarded from the initial sample. To obtain information on individual abundances from the spectral lines of various elements, one must first determine the parameters that characterize the atmospheric model; i.e., the effective temperature $\left(T_{\text {eff }}\right)$, the surface gravity $(\log g)$, the microturbulent velocity $(\xi)$, and the iron abundance. They were calculated in an iterative process from the comparison of the observed spectra and the model for a given set of parameters. The atmospheric parameters and metal abundances were determined using the measured $E W \mathrm{~s}$ and a standard local thermodynamic equilibrium (LTE) analysis with the most recent version of the line abundance code MOOG (Sneden 1973) and a grid of Kurucz (1993) ATLAS9 atmospheres, computed without the overshooting option and with a mixing length to pressure scale height ratio $\alpha=0.5$. 


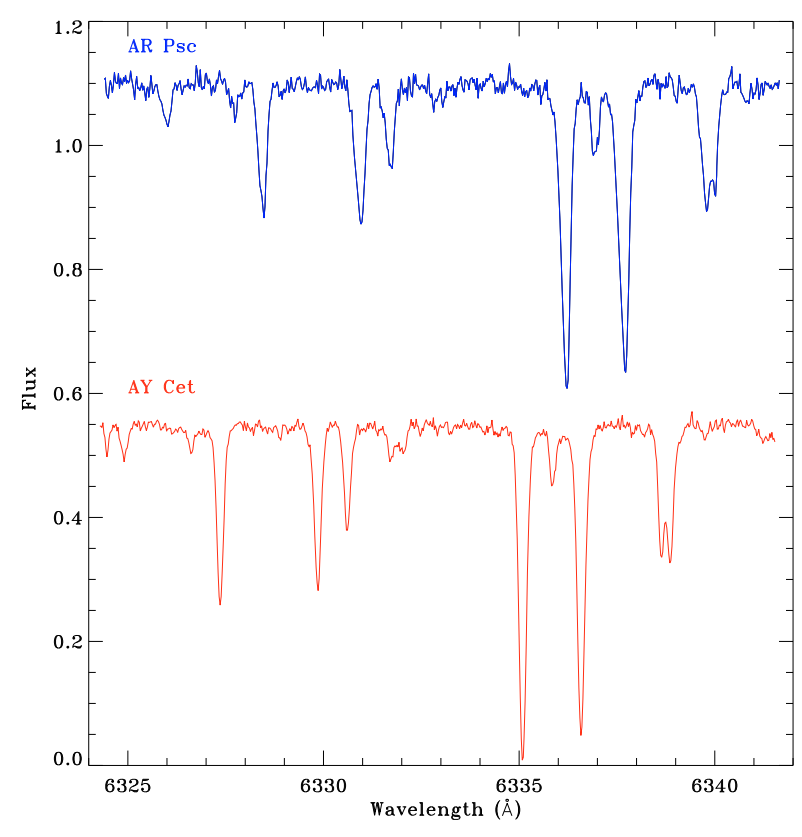

Fig. 3. AR Psc and AY Cet optical spectra.

Assumptions made in the models include: the atmosphere is plane-parallel and in hydrostatic equilibrium, the total flux is constant, the source function is described by the Planck function, the populations of different excitation levels and ionization stages are governed by LTE. The abundances were derived from theoretical curves of growth, computed by MOOG, using model atmospheres and atomic data (wavelength, excitation potential, $g f$ values). The input model was constructed using as atmospheric parameters the average values of previous determinations found in the literature and solar metallicity. Further details on the iterative process followed, and the errors determination is described in Affer et al. (2005). For an elemental abundance derived from many lines, the uncertainty of the atmospheric parameters is the dominant error, while for an abundance derived from a few lines, the uncertainty in the equivalent widths may be more significant. In our case, the atmospheric parameters calculated are $T_{\text {eff }}=4995 \pm 170 \mathrm{~K}, \log g=3.27 \pm 0.60\left[\mathrm{~cm} \mathrm{~s}^{-2}\right]$, and $\xi=1.29 \pm 0.13 \mathrm{~km} \mathrm{~s}^{-1}$ for AR Psc, and $T_{\text {eff }}=4967 \pm 185 \mathrm{~K}$, $\log g=2.34 \pm 0.47\left[\mathrm{~cm} \mathrm{~s}^{-2}\right]$, and $\xi=1.56 \pm 0.08 \mathrm{~km} \mathrm{~s}^{-1}$ for AY Cet. In the case of AY Cet, these parameters are in good agreement with Ottmann et al. (1998) except for lower gravity in our case; iron abundance agrees with that of Ottman et al., but $\mathrm{Mg}$ and $\mathrm{Si}$ are substantially higher $(-0.22$ and -0.32 in their case, respectively).

The coronal abundances are compared to the photosphere of the same stars (Fig. 5), using the solar photospheric values as scale. There is no proof of MAD or any inverse FIP effect in either of the two cases. The best calculated values, the abundance of $\mathrm{Fe}, \mathrm{Ni}$ and $\mathrm{Mg}$, show consistent coronal and photospheric abundances. Some metal depletion seems to take place for $\mathrm{Si}$ and $\mathrm{O}$ in $\mathrm{AY}$ Cet. It must be noticed that photospheric abundances of elements other than $\mathrm{Fe}$ are calculated with fewer lines, so we are less confident about their values. These results confirm those of $\lambda$ And and V851 Cen (Sanz-Forcada et al. 2004), displayed here for easier comparison (Fig. 7, Table 5. It is also worth checking the abundance ratio $[\mathrm{Ne} / \mathrm{O}]$ in the corona. Drake $\&$ Testa (2005) find that an average value of $[\mathrm{Ne} / \mathrm{O}]=0.41$, calculated in the corona of nearby stars, would help for solving the
Table 4. Emission measure distribution of the target stars.

\begin{tabular}{lll}
\hline \hline $\log T$ & \multicolumn{2}{c}{$\log \int N_{\mathrm{e}} N_{\mathrm{H}} \mathrm{d} V\left(\mathrm{~cm}^{-3}\right)^{a}$} \\
$(\mathrm{~K})$ & \multicolumn{1}{c}{ AR Psc } & \multicolumn{1}{c}{ AY Cet } \\
\hline 6.0 & 50.40 & 50.60 \\
6.1 & 50.70 & 50.80 \\
6.2 & $50.90_{-0.30}^{+0.10}$ & $51.10_{-0.40}^{+0.20}$ \\
6.3 & $51.05_{-0.30}^{+0.10}$ & $51.40_{-0.20}^{+0.20}$ \\
6.4 & $51.05_{-0.30}^{+0.20}$ & $51.10_{-0.20}^{+0.30}$ \\
6.5 & $51.20_{-0.30}^{+0.10}$ & $51.55_{-0.30}^{+0.30}$ \\
6.6 & $51.30_{-0.30}^{+0.20}$ & $51.90_{-0.30}^{+0.30}$ \\
6.7 & $51.40_{-0.30}^{+0.20}$ & $52.30_{-0.30}^{+0.20}$ \\
6.8 & $51.70_{-0.20}^{+0.20}$ & $52.85_{-0.20}^{+0.20}$ \\
6.9 & $52.70_{-0.00}^{+0.10}$ & $53.30_{-0.10}^{+0.10}$ \\
7.0 & $52.90_{-0.10}^{+0.00}$ & $53.55_{-0.00}^{+0.00}$ \\
7.1 & $52.40_{-0.20}^{+0.20}$ & $52.40_{-0.25}^{+0.15}$ \\
7.2 & $52.60_{-0.30}^{+0.20}$ & $52.60_{-0.30}^{+0.10}$ \\
7.3 & $52.70_{-0.30}^{+0.10}$ & $52.75_{-0.15}^{+0.15}$ \\
7.4 & $52.50_{-0.30}^{+0.10}$ & $52.70_{-0.25}^{+0.30}$ \\
7.5 & 51.60 & $52.30^{-0.30}$ \\
\hline
\end{tabular}

${ }^{a}$ Emission measure, where $N_{\mathrm{e}}$ and $N_{\mathrm{H}}$ are electron and hydrogen densities, in $\mathrm{cm}^{-3}$. Error bars provided are not independent between the different temperatures, see text.

"solar model problem" (see also discussion in Schmelz et al. 2005). The ratios in the coronae of AR Psc and AY Cet are consistent with the value observed by Drake \& Testa (2005). An upwards correction of the Ne solar abundance, as proposed by these authors, would not affect our results since we compared coronal and photospheric abundances of the same star.

\section{Discussion}

The results are suggestive of a lack of MAD effect. We see once again that coronal abundances of active stars, once they are compared with their own photospheric values instead of the solar photosphere, show no sign of inverse FIP effect or MAD. So far, all the active stars with inverse FIP effect observed (we discard those compared to solar photosphere) have a high projected rotational velocity (Table 1). A large $v \sin i$ broadens the lines observed in the optical wavelengths, those usually employed to calculate photospheric abundances. The broadening of lines might yield erroneous measurements of equivalent widths (blends are included in the measurements, and continuum is more difficult to place), and therefore wrong photospheric abundances. All active stars are fast rotators, but only the cases with high projected rotational velocity will affect the measurements of lines. Ottmann et al. (1998) note that values of $v \sin i \gtrsim 20 \mathrm{~km} \mathrm{~s}^{-1}$ yield wrong results. We therefore are more inclined to believe that the inverse FIP effect is an observational effect, rather than a real fact. Thus, from now on we will interpret the results in this basis, insisting that more observations are needed in order to clarify how real the inverse FIP effect is.

Assuming that no inverse FIP effect exists among active stars, the observations suits the coronal model explained by Laming (2004). The works carried out in the Sun in past years have strengthened the idea of the Alfvén waves as the main force responsible for the energy transportation between chromosphere and corona of the Sun. Recent observations with the mission Hinode (De Pontieu et al. 2007; Erdélyi \& Fedun 2007) support this idea. In the model described by Laming (2004), the Alfvén 

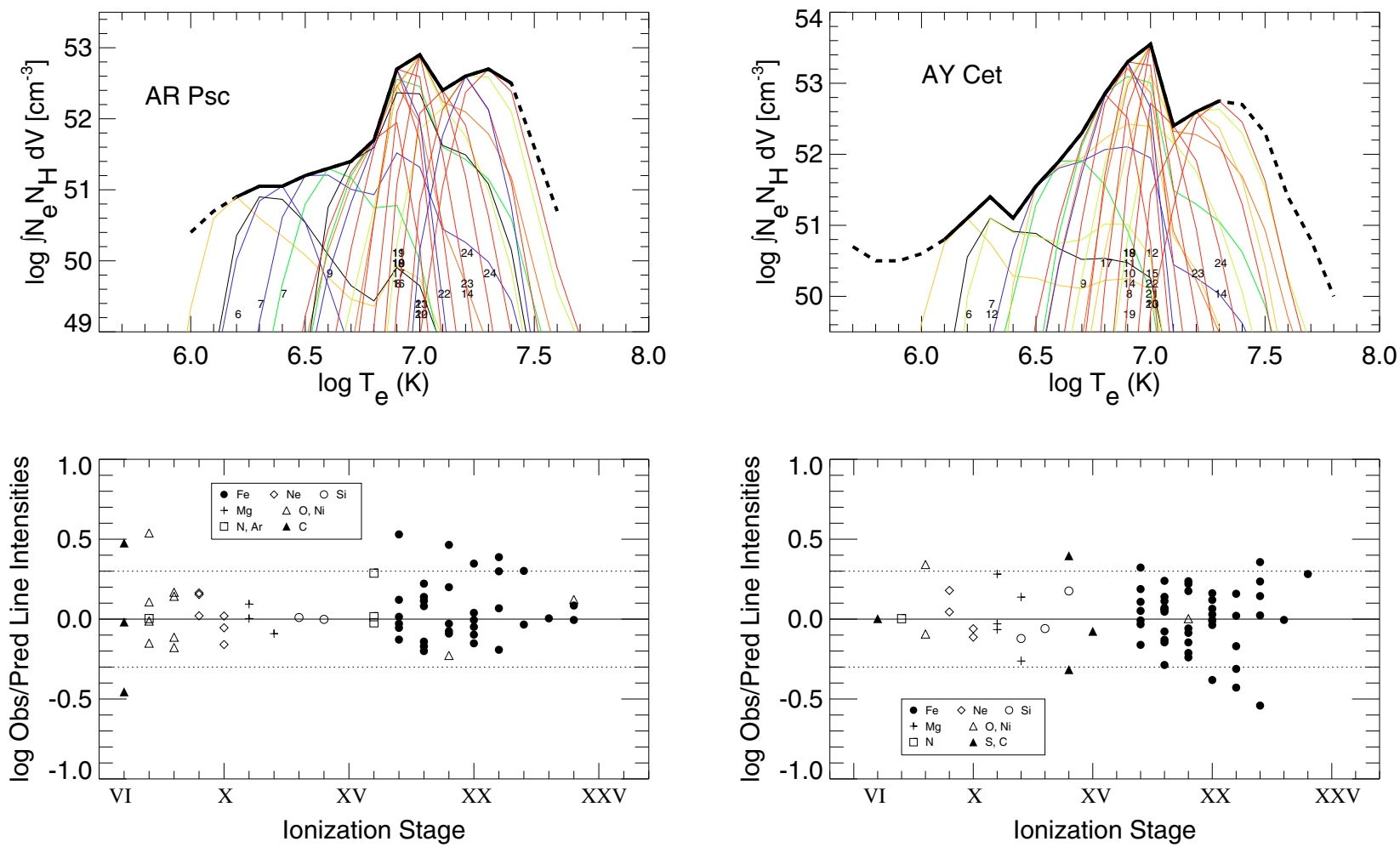

Fig. 4. Emission measure distribution (EMD) of AR Psc and AY Cet. Thin lines represent the relative contribution function for each ion (the emissivity function multiplied by the EMD at each point). Small numbers indicate the ionization stages of the species. Also plotted are the observed-to-predicted line flux ratios for the ion stages in the upper figure. The dotted lines denote a factor of 2.
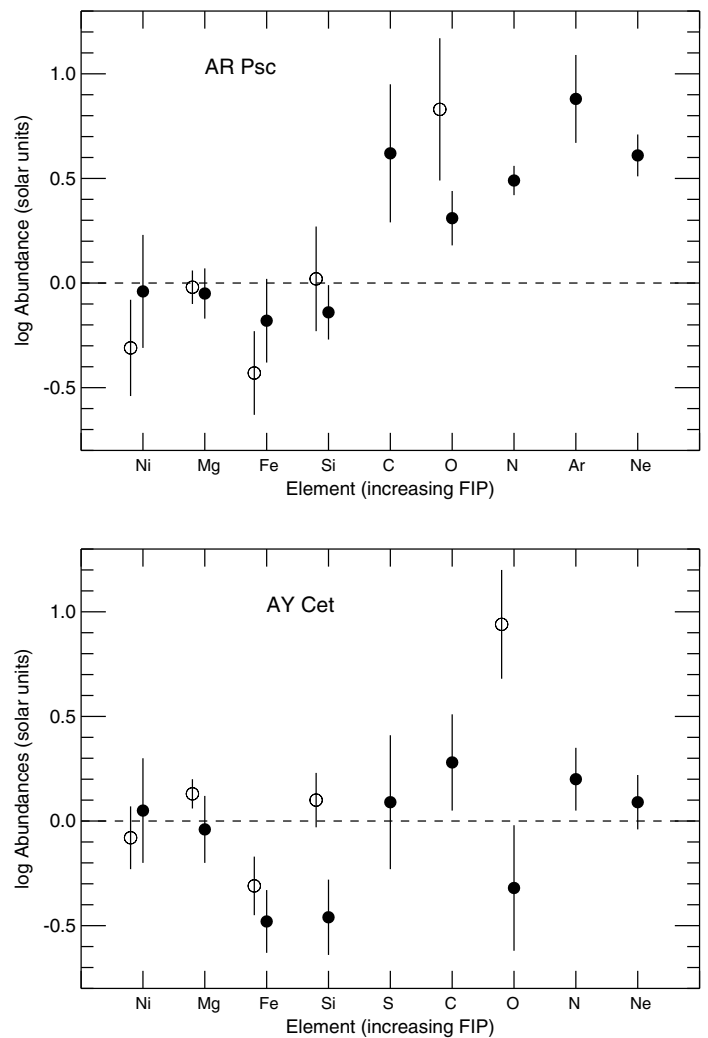

Fig. 5. Coronal abundances of AR Psc and AY Cet, in increasing order of FIP. Filled circles are coronal values and open circles represent photospheric values. A dashed line indicates the adopted solar photospheric abundance (Asplund et al. 2005).
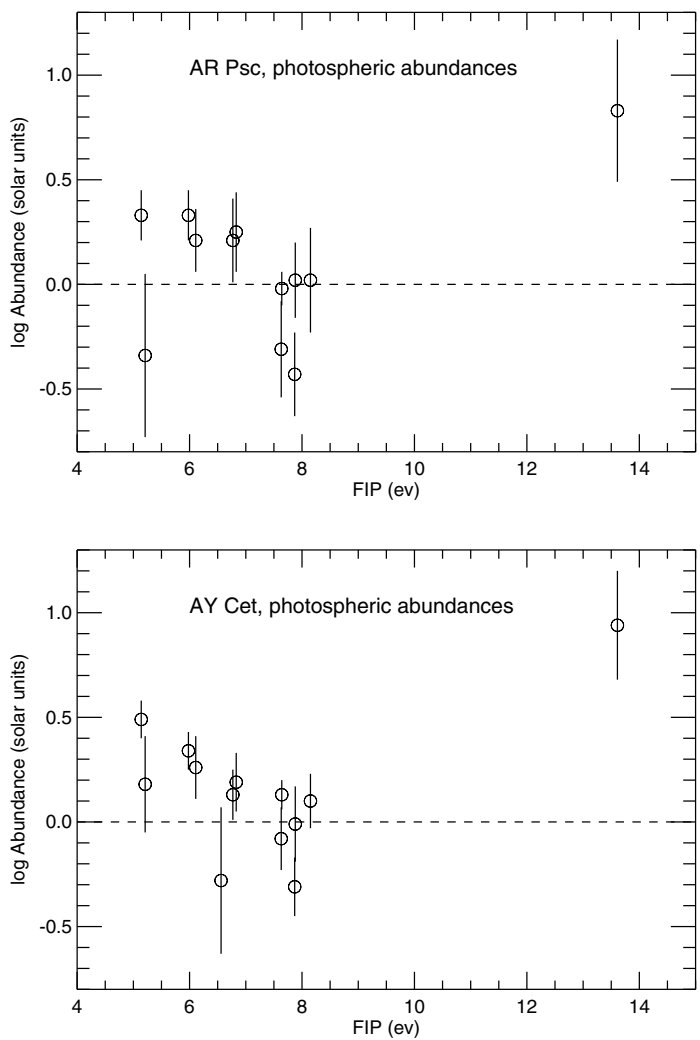

Fig. 6. Photospheric abundances of AR Psc and AY Cet. A dashed line indicates the adopted solar photospheric abundance (Asplund et al. 2005). 

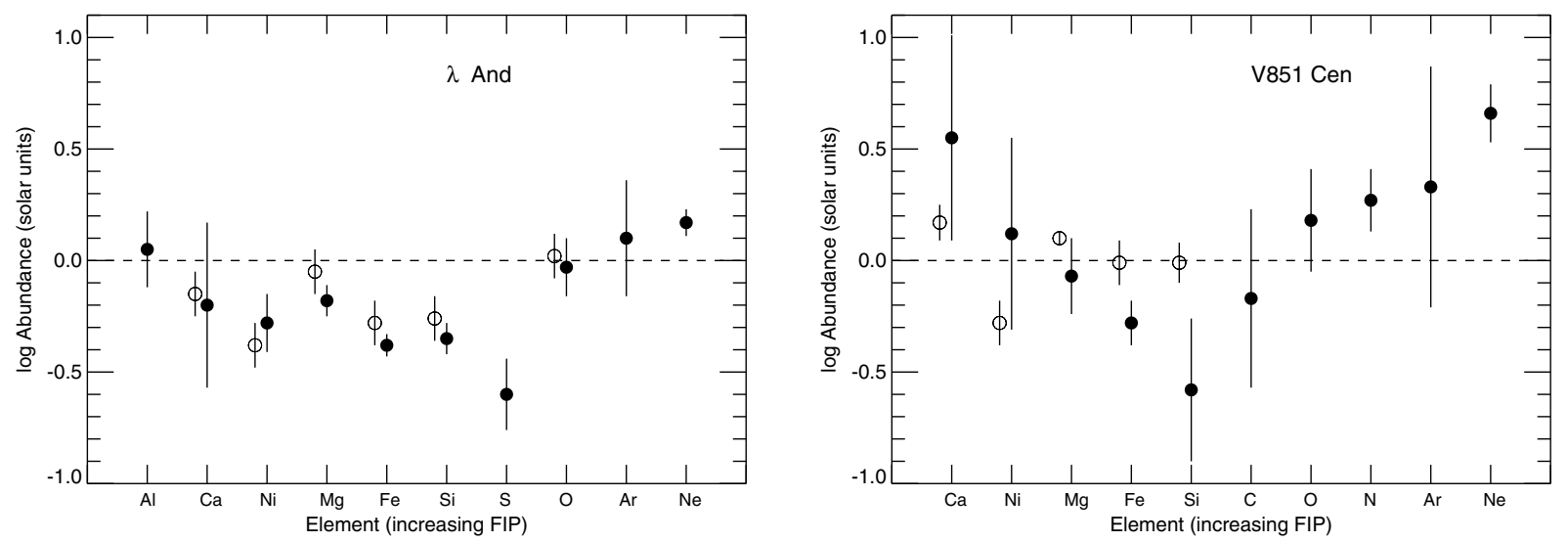

Fig. 7. Same as in Fig. 5, but for $\lambda$ And and V851 Cen. Results from Sanz-Forcada et al. (2004), adapted to the solar reference values of Asplund et al. (2005).

Table 5. Coronal and photospheric abundances of the elements $([\mathrm{X} / \mathrm{H}]$, solar units) in the target stars.

\begin{tabular}{lrlrrrrrrrrr}
\hline \hline $\mathrm{X}$ & $\begin{array}{r}\text { FIP } \\
\text { eV }\end{array}$ & \multicolumn{2}{c}{ Ref. $^{a}$} & $\begin{array}{c}\text { solar pho }^{a} \text { ) } \\
\end{array}$ & \multicolumn{2}{c}{ AR Psc } & \multicolumn{4}{c}{ AY Cet } & \multicolumn{2}{c}{$\lambda$ And $^{b}$} & \multicolumn{3}{c}{ V851 Cen $^{b}$} \\
Photosphere & Corona & Photosphere & Corona & Photosphere & Corona & Photosphere & Corona \\
\hline $\mathrm{Na}$ & 5.14 & 6.17 & $(6.33)$ & $0.33 \pm 0.12$ & $\ldots$ & $0.49 \pm 0.09$ & $\ldots$ & $-0.09 \pm 0.10$ & $\ldots$ & $0.39 \pm 0.11$ \\
$\mathrm{Al}$ & 5.98 & 6.37 & $(6.47)$ & $0.33 \pm 0.12$ & $\ldots$ & $0.34 \pm 0.09$ & $\ldots$ & $\ldots$ & $0.05 \pm 0.17$ & $0.35 \pm 0.05$ & $\ldots$ \\
$\mathrm{Ca}$ & 6.11 & 6.31 & $(6.36)$ & $0.21 \pm 0.15$ & $\ldots$ & $0.26 \pm 0.15$ & $\ldots$ & $-0.15 \pm 0.10$ & $-0.20 \pm 0.37$ & $0.17 \pm 0.08$ & $0.55 \pm 0.46$ \\
$\mathrm{Ni}$ & 7.63 & 6.23 & $(6.25)$ & $-0.31 \pm 0.23$ & $-0.04 \pm 0.27$ & $-0.08 \pm 0.15$ & $0.05 \pm 0.25$ & $-0.38 \pm 0.10$ & $-0.28 \pm 0.13$ & $-0.28 \pm 0.10$ & $0.12 \pm 0.43$ \\
$\mathrm{Mg}$ & 7.64 & 7.53 & $(7.58)$ & $-0.02 \pm 0.08$ & $-0.05 \pm 0.12$ & $0.13 \pm 0.07$ & $-0.04 \pm 0.16$ & $-0.05 \pm 0.10$ & $-0.18 \pm 0.07$ & $0.10 \pm 0.03$ & $-0.07 \pm 0.17$ \\
$\mathrm{Fe}$ & 7.87 & 7.45 & $(7.67)$ & $-0.43 \pm 0.20$ & $-0.18 \pm 0.20$ & $-0.31 \pm 0.14$ & $-0.48 \pm 0.15$ & $-0.28 \pm 0.10$ & $-0.38 \pm 0.05$ & $-0.01 \pm 0.10$ & $-0.28 \pm 0.10$ \\
$\mathrm{Si}$ & 8.15 & 7.51 & $(7.55)$ & $0.02 \pm 0.25$ & $-0.14 \pm 0.13$ & $0.10 \pm 0.13$ & $-0.46 \pm 0.18$ & $-0.26 \pm 0.10$ & $-0.35 \pm 0.07$ & $-0.01 \pm 0.09$ & $-0.58 \pm 0.32$ \\
$\mathrm{~S}$ & 10.36 & 7.14 & $(7.21)$ & $\ldots$ & $\ldots$ & $\ldots$ & $0.09 \pm 0.32$ & $\ldots$ & $-0.60 \pm 0.16$ & $\ldots$ & $-0.98 \pm 1.32$ \\
$\mathrm{C}$ & 11.26 & 8.39 & $(8.56)$ & $\ldots$ & $0.62 \pm 0.33$ & $\ldots$ & $0.28 \pm 0.23$ & $\ldots$ & $\ldots$ & $\ldots$ & $-0.17 \pm 0.40$ \\
$\mathrm{O}$ & 13.61 & 8.66 & $(8.93)$ & $0.83 \pm 0.34$ & $0.31 \pm 0.13$ & $0.94 \pm 0.26$ & $-0.32 \pm 0.30$ & $0.02 \pm 0.10$ & $-0.03 \pm 0.13$ & $\ldots$ & $0.18 \pm 0.23$ \\
$\mathrm{~N}$ & 14.53 & 7.78 & $(8.05)$ & $\ldots$ & $0.49 \pm 0.07$ & $\ldots$ & $0.20 \pm 0.15$ & $\ldots$ & $\ldots$ & $\ldots$ & $0.27 \pm 0.14$ \\
$\mathrm{Ar}$ & 15.76 & 6.18 & $(6.56)$ & $\ldots$ & $0.88 \pm 0.21$ & $\ldots$ & $\ldots$ & $\ldots$ & $0.10 \pm 0.26$ & $\ldots$ & $0.33 \pm 0.54$ \\
$\mathrm{Ne}$ & 21.56 & 7.84 & $(8.09)$ & $\ldots$ & $0.61 \pm 0.10$ & $\ldots$ & $0.09 \pm 0.13$ & $\ldots$ & $0.17 \pm 0.06$ & $\ldots$ & $0.66 \pm 0.13$ \\
\hline
\end{tabular}

${ }^{a}$ Solar photospheric abundances from Asplund et al. (2005), adopted in this work, are expressed in logarithmic scale. Note that several values have been updated in the literature since Anders \& Grevesse (1989), also listed in parenthesis for easier comparison.

${ }^{b}$ Results adapted from Sanz-Forcada et al. (2004).

waves, combined with pondermotive forces, would be responsible for part of the energy transportation in the corona.

Their model explains the observed abundances pattern in the solar corona: either the low FIP in the coronal active regions and the slow wind or the absence of FIP effect in coronal holes or fast wind. According to this model, the stars earlier than the Sun, like Procyon, would behave more like coronal holes, because they have shallower convective zones yielding smaller coronal magnetic fields. Their chromospheric Alfvén waves are stronger than the coronal fields, similar to a coronal hole. In stars later than the Sun, but not very active yet, there is a deeper convection, yielding lower frequency chromospheric waves and higher coronal magnetic fields. The wave transmission is less efficient and this would yield a reduced FIP effect. This is the case for $\epsilon$ Eri or $\alpha$ Cen.

Following the same reasoning, it makes sense to find that Alfvén waves are more inefficient for the more active stars, yielding no FIP fractionation for stars such as AR Psc or AY Cet. Some arguments have been proposed by Laming (2004) to explain why an inverse FIP effect could be present in active stars, such as AB Dor: wave reflection in the chromosphere (more likely for stars with lower gravity), or turbulence introduced by differential rotation.
A more complete census of active stars with well measured photospheric and coronal abundances would be necessary to confirm the results, but if we assume that the cases with inverse FIP effect observed are a product of observational effects, the model proposed by Laming (2004) could actually explain the observed abundances patterns in both low and high activity stars.

\section{Conclusions}

The results suggest that metal abundance depletion, or inverse FIP effect, are not present in these two stars. This agrees with results for other active stars with well-known photospheric abundances, such as $\lambda$ And or V851 Cen. Stars with observed inverse FIP effect have their photospheric lines broadened by the high rotation of the stars, hampering their photospheric measurements. The results observed in AR Psc and AY Cet suit the expected behavior in active stars according to the model of Laming (2004), which combines the use of pondermotive forces with Alfvén waves. The same model is also able to explain both the FIP effect in the solar corona and slow wind and the absence of FIP fractionation in solar coronal holes and fast wind. Further measurements in active stars with low projected rotational velocity would help to assure this confirmation of the model. 
Acknowledgements. We acknowledge support by the Ramón y Cajal Program ref. RYC-2005-000549, financed by the Spanish Ministry of Science. J.S. wants to thank Aitor Ibarra for his aid in the treatment of XMM data. This research has also made use of NASA's Astrophysics Data System Abstract Service and the SIMBAD Database, operated at CDS, Strasbourg (France).

\section{References}

Affer, L., Micela, G., Morel, T., Sanz-Forcada, J., \& Favata, F. 2005, A\&A, 433, 647

Anders, E., \& Grevesse, N. 1989, Geochim. Cosmochim. Acta, 53, 197

Argiroffi, C., Maggio, A., \& Peres, G. 2003, A\&A, 404, 1033

Asplund, M., Grevesse, N., \& Sauval, A. J. 2005, in Cosmic Abundances as Records of Stellar Evolution and Nucleosynthesis, ed. T. G. Barnes, III, \& F. N. Bash, ASP Conf. Ser., 336, 25

Audard, M., Güdel, M., Sres, A., Raassen, A. J. J., \& Mewe, R. 2003, A\&A 398, 1137

Audard, M., Telleschi, A., Güdel, M., et al. 2004, ApJ, 617, 531

Ball, B., Drake, J. J., Lin, L., et al. 2005, ApJ, 634, 1336

Brickhouse, N. S., Dupree, A. K., Edgar, R. J., et al. 2000, ApJ, 530, 387

Brinkman, A. C., Behar, E., Güdel, M., et al. 2001, A\&A, 365, L324

De Pontieu, B., McIntosh, S. W., Carlsson, M., et al. 2007, Science, 318, 1574

den Herder, J. W., Brinkman, A. C., Kahn, S. M., et al. 2001, A\&A, 365, L7

Drake, J. J., \& Testa, P. 2005, Nature, 436, 525

Drake, J. J., Laming, J. M., \& Widing, K. G. 1997, ApJ, 478, 403

Erdélyi, R., \& Fedun, V. 2007, Science, 318, 1572

Favata, F., \& Micela, G. 2003, Space Sci. Rev., 108, 577

Feldman, U., \& Laming, J. M. 2000, Phys. Scr., 61, 222

García-Alvarez, D., Drake, J. J., Lin, L., Kashyap, V. L., \& Ball, B. 2005, ApJ, 621, 1009

García-Alvarez, D., Drake, J. J., Kashyap, V. L., Lin, L., \& Ball, B. 2008, ApJ, 679,1509

Güdel, M. 2004, A\&ARv, 12, 71
Huenemoerder, D. P., Canizares, C. R., \& Schulz, N. S. 2001, ApJ, 559, 1135 Huenemoerder, D. P., Canizares, C. R., Drake, J. J., \& Sanz-Forcada, J. 2003, ApJ, 595, 1131

Kurucz, R. L. 1993, SYNTHE spectrum synthesis programs and line data, ed. R. L. Kurucz

Laming, J. M. 2004, ApJ, 614, 1063

Laming, J. M., Drake, J. J., \& Widing, K. G. 1995, ApJ, 443, 416

Laming, J. M., Drake, J. J., \& Widing, K. G. 1996, ApJ, 462, 948

Massarotti, A., Latham, D. W., Stefanik, R. P., \& Fogel, J. 2008, AJ, 135, 209

McWilliam, A. 1990, ApJS, 74, 1075

Montes, D., Fernandez-Figueroa, M. J., de Castro, E., \& Sanz-Forcada, J. 1997, A\&AS, 125, 263

Morel, T., Micela, G., Favata, F., Katz, D., \& Pillitteri, I. 2003, A\&A, 412, 495

Ness, J.-U., \& Jordan, C. 2008, MNRAS, 385, 1691

Nordström, B., Mayor, M., Andersen, J., et al. 2004, A\&A, 418, 989

Ottmann, R., Pfeiffer, M. J., \& Gehren, T. 1998, A\&A, 338, 661

Raassen, A. J. J., Mewe, R., Audard, M., et al. 2002, A\&A, 389, 228

Raassen, A. J. J., Ness, J.-U., Mewe, R., et al. 2003, A\&A, 400, 671

Randich, S., Gratton, R., \& Pallavicini, R. 1993, A\&A, 273, 194

Sanz-Forcada, J., Brickhouse, N. S., \& Dupree, A. K. 2003a, ApJS, 145, 147

Sanz-Forcada, J., Maggio, A., \& Micela, G. 2003b, A\&A, 408, 1087

Sanz-Forcada, J., Favata, F., \& Micela, G. 2004, A\&A, 416, 281

Schmelz, J. T., Nasraoui, K., Roames, J. K., Lippner, L. A., \& Garst, J. W. 2005, ApJ, 634, L197

Schmitt, J. H. M. M., Stern, R. A., Drake, J. J., \& Kuerster, M. 1996, ApJ, 464, 898

Shan, H., Liu, X., \& Gu, S. 2006, New Astron., 11, 287

Smith, R. K., Brickhouse, N. S., Liedahl, D. A., \& Raymond, J. C. 2001, ApJ, 556, L91

Sneden, C. A. 1973, Ph.D. thesis, The University of Texas at Austin

Suh, J. A., Audard, M., Güdel, M., \& Paerels, F. B. S. 2005, ApJ, 630, 1074

Telleschi, A., Güdel, M., Briggs, K., et al. 2005, ApJ, 622, 653

Weisskopf, M. C., Brinkman, B., Canizares, C., et al. 2002, PASP, 114, 1

Wood, B. E., \& Linsky, J. L. 2006, ApJ, 643, 444 
J. Sanz-Forcada et al.: No first ionization potential fractionation in the active stars AR Piscium and AY Ceti, Online Material $p 1$

Table 2. XMM/RGS line fluxes of $\mathrm{AR} \mathrm{Psc}^{a}$.

\begin{tabular}{|c|c|c|c|c|c|c|}
\hline Ion & $\lambda_{\text {model }}$ & $\log T_{\max }$ & $F_{\mathrm{obs}}$ & $S / N$ & Ratio & Blends \\
\hline Si XIV & 6.1804 & 7.2 & $8.06 \mathrm{e}-14$ & 4.3 & -0.00 & \\
\hline Si XIII & 6.6479 & 7.0 & $2.43 \mathrm{e}-13$ & 10.2 & 0.01 & Si XIII 6.6882, 6.7403 \\
\hline Mg XII & 8.4192 & 7.0 & $1.59 \mathrm{e}-13$ & 5.9 & -0.09 & Mg XII 8.4246 \\
\hline $\mathrm{Mg}$ XI & 9.1687 & 6.8 & $1.12 \mathrm{e}-13$ & 10.8 & 0.00 & Mg XI 9.2312 \\
\hline Mg XI & 9.3143 & 6.8 & $5.11 \mathrm{e}-14$ & 7.5 & 0.09 & Ni XXV 9.3400, Fe XXII 9.3933 \\
\hline $\mathrm{Fe} X \mathrm{XI}$ & 9.4797 & 7.0 & $1.15 \mathrm{e}-13$ & 11.5 & 0.30 & Ne X 9.4807, 9.4809, Ni XXVI 9.5292, Fe XXI 9.5443 \\
\hline $\mathrm{Ne} \mathrm{x}$ & 9.7080 & 6.8 & $4.34 \mathrm{e}-14$ & 7.2 & -0.16 & Ne X 9.7085, Fe Xx 9.7269 \\
\hline $\mathrm{Fe} x \mathrm{x}$ & 10.1203 & 7.0 & $5.74 \mathrm{e}-14$ & 8.6 & 0.35 & Fe XX $10.0529,10.0596$, Ni XIX 10.1100, Fe XIX 10.1419 \\
\hline $\operatorname{Nex}$ & 10.2385 & 6.8 & $1.55 \mathrm{e}-13$ & 8.2 & 0.02 & Ne $x 10.2396$ \\
\hline Ni XXIV & 10.2770 & 7.2 & $1.23 \mathrm{e}-14$ & 4.1 & 0.12 & Fe XX 10.2675 \\
\hline Fe XXIV & 10.6190 & 7.3 & $8.69 \mathrm{e}-14$ & 11.3 & -0.01 & Fe XIX 10.6414, 10.6491, Fe XXIV 10.6630 \\
\hline Fe XIX & 10.8160 & 6.9 & $2.33 \mathrm{e}-14$ & 5.9 & -0.08 & Ne IX 10.7650, Fe XVII 10.7700 \\
\hline Fe XXIII & 10.9810 & 7.2 & $1.11 \mathrm{e}-13$ & 13.1 & 0.00 & Ne IX 11.0010, Fe XXIII 11.0190, Fe XXIV 11.0290 \\
\hline Fe Xxiv & 11.1760 & 7.3 & $7.44 \mathrm{e}-14$ & 10.9 & 0.09 & Fe XVII 11.1310, Fe XXIV 11.1870 \\
\hline Fe XVII & 11.2540 & 6.8 & $2.22 \mathrm{e}-14$ & 6.0 & -0.05 & Fe XXIV 11.2680, Fe XXIII 11.2850 \\
\hline Fe XVIII & 11.4230 & 6.9 & $4.36 \mathrm{e}-14$ & 3.4 & -0.17 & Fe XXII 11.4270 , Fe XXIV 11.4320 , Fe XXIII 11.4580 \\
\hline $\mathrm{Ne} I X$ & 11.5440 & 6.6 & $9.44 \mathrm{e}-14$ & 12.1 & 0.15 & Fe XXII 11.4900, Fe XVIII 11.5270, Ni XIX 11.5390 \\
\hline Fe XXII & 11.7700 & 7.1 & $1.86 \mathrm{e}-13$ & 9.4 & -0.03 & Fe XXIII 11.7360 \\
\hline Fe XXII & 11.9770 & 7.1 & $1.25 \mathrm{e}-13$ & 5.7 & 0.30 & Fe XXII 11.8810, 11.9320, Fe XXIII 11.8980, Fe XXI 11.9466, 11.9750 \\
\hline $\mathrm{Ne} \mathrm{X}$ & 12.1321 & 6.8 & $8.36 \mathrm{e}-13$ & 19.6 & -0.05 & NeX 12.1375 \\
\hline Fe XXI & 12.2840 & 7.0 & $1.31 \mathrm{e}-13$ & 7.7 & -0.19 & Fe XVII 12.2660 \\
\hline Ni XIX & 12.4350 & 6.9 & $5.83 \mathrm{e}-14$ & 10.6 & -0.23 & Fe XXI 12.3930, 12.4220, Fe XXII 12.4311, 12.4318 \\
\hline Fe XXI & 12.4990 & 7.0 & $5.01 \mathrm{e}-14$ & 3.6 & 0.39 & Ni XXI 12.5105, Fe XX 12.5260 \\
\hline $\mathrm{Fe} \mathrm{XX}$ & 12.8460 & 7.0 & $1.73 \mathrm{e}-13$ & 10.9 & -0.15 & Fe XXI 12.8220, Fe XX $12.8240,12.8640$ \\
\hline $\mathrm{Fe} \mathrm{xx}$ & 12.9650 & 7.0 & $8.99 \mathrm{e}-14$ & 12.9 & -0.10 & Fe XX 12.9120, 12.9920, 13.0240, Fe XIX 12.9330, 13.0220, Fe XXII 12.9530 \\
\hline $\mathrm{Fe} x \mathrm{x}$ & 13.1530 & 7.0 & $4.12 \mathrm{e}-14$ & 8.5 & -0.05 & Fe XX 13.1370, Fe XXI 13.1155, 13.1671, 13.1678 \\
\hline $\mathrm{Fe} \mathrm{xx}$ & 13.2740 & 7.0 & $3.75 \mathrm{e}-14$ & 3.1 & -0.01 & Fe XX 13.2909, Fe XXII 13.2360, Fe XXI 13.2487 , Ni XX 13.2560, Fe XIX 13.2658 \\
\hline $\mathrm{Fe} x \mathrm{x}$ & 13.3089 & 7.0 & $1.88 \mathrm{e}-14$ & 6.2 & 0.04 & Ni XX 13.3090, Fe XIX 13.3191, Fe XVIII 13.3230 \\
\hline $\mathrm{Ne} I \mathrm{X}$ & 13.4473 & 6.6 & $2.47 \mathrm{e}-13$ & 9.6 & 0.02 & Fe XX 13.3850, Fe XIX 13.4620 \\
\hline Fe XIX & 13.5180 & 6.9 & $1.74 \mathrm{e}-13$ & 19.2 & -0.09 & Fe XIX 13.4970, Fe XXI 13.5070, Ne IX 13.5531 \\
\hline Ne IX & 13.6990 & 6.6 & $1.69 \mathrm{e}-13$ & 19.0 & 0.16 & Fe XIX 13.6450, 13.7315, 13.7458 \\
\hline Fe XIX & 13.7950 & 6.9 & $7.97 \mathrm{e}-14$ & 13.1 & -0.03 & Fe XX 13.7670, Ni XIX 13.7790, Fe XVII 13.8250 \\
\hline Fe XXI & 14.0080 & 7.0 & $9.01 \mathrm{e}-14$ & 13.9 & 0.07 & Ni XIX 14.0430, 14.0770, Fe XIX 14.0717 \\
\hline Fe XVIII & 14.2080 & 6.9 & $1.31 \mathrm{e}-13$ & 16.8 & -0.14 & Fe XVIII 14.2560, Fe XX 14.2670 \\
\hline Fe XVIII & 14.3730 & 6.9 & $1.19 \mathrm{e}-13$ & 6.7 & 0.08 & Fe Xx 14.3318, 14.4207, 14.4600, Fe XVIII 14.3430, 14.4250 \\
\hline Fe XVIII & 14.5340 & 6.9 & $6.86 \mathrm{e}-14$ & 4.8 & 0.14 & Fe XVIII $14.4856,14.5056,14.5710,14.6011$, Fe XX 14.5146 \\
\hline Fe XIX & 14.6640 & 6.9 & $3.92 \mathrm{e}-14$ & 9.1 & 0.20 & Fe XVIII 14.6884 \\
\hline O VIII & 14.8205 & 6.5 & $9.55 \mathrm{e}-14$ & 14.4 & 0.17 & Fe XIX 14.7250, Fe XX 14.7540, 14.8526, Fe XVIII 14.7820, O VIII 14.8207 \\
\hline FexVII & 15.0140 & 6.7 & $1.85 \mathrm{e}-13$ & 12.3 & -0.13 & \\
\hline O VIII & 15.1760 & 6.5 & $1.46 \mathrm{e}-13$ & 10.1 & 0.14 & Fe XIX 15.0790, 15.1980, O VIII 15.1765 \\
\hline FeXVII & 15.2610 & 6.7 & $7.26 \mathrm{e}-14$ & 6.4 & 0.12 & \\
\hline Fe XVII & 15.4530 & 6.7 & $5.98 \mathrm{e}-14$ & 6.2 & 0.53 & Fe XX 15.4077, 15.5170, Fe XIX 15.4136, 15.4655, Fe XVIII 15.4940, 15.5199 \\
\hline Fe XVIII & 15.8240 & 6.8 & $4.61 \mathrm{e}-14$ & 3.7 & 0.22 & Fe XVIII 15.8700 \\
\hline O VIII & 16.0055 & 6.5 & $1.36 \mathrm{e}-13$ & 10.0 & -0.18 & Fe XVIII 16.0040, O VIII 16.0067 \\
\hline Fe XVIII & 16.0710 & 6.8 & $5.72 \mathrm{e}-14$ & 4.9 & -0.20 & Fe XIX 16.1100, Fe XVIII 16.1590 \\
\hline Fe XIX & 16.2830 & 6.9 & $5.28 \mathrm{e}-14$ & 6.4 & 0.46 & Fe XVIII 16.3200, Fe XIX 16.3414, Fe XVII 16.3500 \\
\hline Fe XVII & 16.7800 & 6.7 & $6.91 \mathrm{e}-14$ & 12.6 & -0.03 & \\
\hline Fe XVII & 17.0510 & 6.7 & $1.71 \mathrm{e}-13$ & 19.7 & 0.01 & Fe XVII 17.0960 \\
\hline Fe XVIII & 17.6230 & 6.8 & $3.38 \mathrm{e}-14$ & 4.6 & 0.11 & \\
\hline OVII & 17.7680 & 6.4 & $3.95 \mathrm{e}-14$ & 9.3 & 0.54 & ArXVI $17.7320,17.7420$ \\
\hline OVII & 18.6270 & 6.3 & $2.78 \mathrm{e}-14$ & 7.9 & -0.01 & Ar XVI 18.6240, Ca XVIII 18.6910 \\
\hline O VIII & 18.9671 & 6.5 & $6.91 \mathrm{e}-13$ & 39.9 & -0.11 & O VIII 18.9725 \\
\hline O VII & 21.6015 & 6.3 & $6.11 \mathrm{e}-14$ & 11.3 & -0.15 & \\
\hline O VII & 22.0977 & 6.3 & $6.61 \mathrm{e}-14$ & 11.7 & 0.11 & Ca XVII 22.1140 \\
\hline Ar XVI & 23.5460 & 6.7 & $2.92 \mathrm{e}-14$ & 4.3 & 0.01 & Ar XVI 23.5900 \\
\hline N VII & 24.7792 & 6.3 & $8.23 \mathrm{e}-14$ & 13.8 & 0.00 & N VII 24.7846 \\
\hline ArXVI & 24.8540 & 6.7 & $2.60 \mathrm{e}-14$ & 3.4 & 0.29 & \\
\hline Ar XVI & 24.9910 & 6.7 & $2.83 \mathrm{e}-14$ & 7.9 & -0.02 & Ar XVI 25.0130, Ar XV 25.0500 \\
\hline C VI & 26.9896 & 6.2 & $4.11 \mathrm{e}-14$ & 5.7 & 0.48 & C VI 26.9901 \\
\hline $\mathrm{CVI}$ & 28.4652 & 6.2 & $4.33 e-14$ & 4.6 & -0.02 & Ar XV 28.3860, C VI 28.4663 \\
\hline C VI & 33.7342 & 6.1 & $7.86 \mathrm{e}-14$ & 6.3 & -0.45 & C VI 33.7396 \\
\hline
\end{tabular}

${ }^{a}$ Line fluxes (in erg $\mathrm{cm}^{-2} \mathrm{~s}^{-1}$ ) measured in XMM/RGS AR Psc spectra, and corrected by the ISM absorption. $\log T_{\max }$ indicates the maximum temperature $(\mathrm{K})$ of formation of the line (unweighted by the EMD). "Ratio" is the $\log \left(F_{\mathrm{obs}} / F_{\text {pred }}\right)$ of the line. Blends amounting to more than $5 \%$ of the total flux for each line are indicated. 
Table 3. Chandra/LETG line fluxes of AY Cet ${ }^{a}$.

\begin{tabular}{|c|c|c|c|c|c|c|}
\hline Ion & $\lambda_{\text {model }}$ & $\log T_{\max }$ & $F_{\mathrm{obs}}$ & $S / N$ & Ratio & Blends \\
\hline $\mathrm{SXV}$ & 5.1015 & 7.2 & $9.72 \mathrm{e}-14$ & 3.3 & -0.08 & S XV 5.0387 \\
\hline Si XIV & 6.1804 & 7.2 & $5.86 \mathrm{e}-14$ & 5.4 & 0.18 & Si XIV 6.1858 \\
\hline Si XIII & 6.6479 & 7.0 & $1.11 \mathrm{e}-13$ & 7.9 & -0.06 & Si XIII $6.6882,6.7403$ \\
\hline Mg XII & 7.1058 & 7.0 & $4.21 \mathrm{e}-14$ & 4.9 & 0.14 & Mg XII 7.1069, Al XIII 7.1710 \\
\hline Mg XI & 7.8503 & 6.8 & $4.20 \mathrm{e}-14$ & 4.9 & 0.28 & $\mathrm{Al}$ XII 7.8721 \\
\hline Mg XII & 8.4192 & 7.0 & $9.72 \mathrm{e}-14$ & 7.8 & -0.26 & Mg XII 8.4246 \\
\hline Mg XI & 9.1687 & 6.8 & $1.08 \mathrm{e}-13$ & 7.9 & -0.07 & \\
\hline Mg XI & 9.3143 & 6.8 & $7.06 \mathrm{e}-14$ & 6.4 & -0.03 & Mg XI 9.2312, Ni XIX 9.2540 \\
\hline $\mathrm{Fe} \mathrm{XX}$ & 9.9977 & 7.0 & $2.48 \mathrm{e}-14$ & 3.8 & -0.04 & Ni XIX 9.9770, Fe XXI 9.9887, Fe XX 10.0004, 10.0054 \\
\hline Nex & 10.2385 & 6.8 & $4.17 \mathrm{e}-14$ & 4.9 & -0.11 & Nex 10.2396 \\
\hline Fe XIX & 10.6414 & 6.9 & $3.10 \mathrm{e}-14$ & 4.2 & -0.09 & Fe XVIII 10.5364, Fe XXIV 10.6190, 10.6630, Fe XIX 10.6295, 10.6491, 10.6840, Fe XVII 10.6570 \\
\hline Fe XIX & 10.8160 & 6.9 & $2.07 \mathrm{e}-14$ & 3.5 & 0.18 & Fe XVII 10.7700 \\
\hline Fe XXIII & 10.9810 & 7.2 & $3.16 \mathrm{e}-14$ & 4.3 & -0.01 & Ne IX 11.0010, Fe XXIII 11.0190, Fe XVII 11.0260, Fe XXIV 11.0290 \\
\hline Fe XXIV & 11.1760 & 7.3 & $7.50 \mathrm{e}-14$ & 6.8 & 0.28 & Fe XVII 11.1310, Ni XXII 11.1818, 11.1950, 11.2118 \\
\hline Fe XVIII & 11.3260 & 6.9 & $3.83 \mathrm{e}-14$ & 4.9 & 0.11 & Fe XVII 11.2540, Ni XXI 11.3180 \\
\hline Fe XVIII & 11.4230 & 6.9 & $2.61 \mathrm{e}-14$ & 4.1 & 0.07 & Fe XXII 11.4270, Fe XXIV 11.4320, Fe XVIII 11.4494 \\
\hline Fe XVIII & 11.5270 & 6.9 & $4.42 \mathrm{e}-14$ & 5.4 & 0.05 & Fe XXII 11.4900 , Ni XIX 11.5390 , Ni XXI 11.5390 , Ne IX 11.5440 \\
\hline Fe XXII & 11.7700 & 7.1 & $9.48 \mathrm{e}-14$ & 7.9 & 0.02 & Fe XXIII 11.7360, Ni XX 11.8320, 11.8460 \\
\hline Fe XXII & 11.9770 & 7.1 & $8.26 \mathrm{e}-14$ & 7.5 & 0.36 & Fe XXII 11.8810, 11.9320, Ni XX 11.9617, Fe XXI 11.9750, 12.0440 \\
\hline $\mathrm{Ne} \mathrm{x}$ & 12.1321 & 6.8 & $3.17 \mathrm{e}-13$ & 14.7 & -0.06 & Fe XVII 12.1240, Ne X 12.1375 \\
\hline Fe XXI & 12.2840 & 7.0 & $1.57 \mathrm{e}-13$ & 10.4 & 0.16 & Fe XXII 12.2100, Fe XVII 12.2660 \\
\hline Fe XXI & 12.3930 & 7.0 & $1.05 \mathrm{e}-13$ & 8.5 & 0.02 & Fe XXI 12.4220, Ni XIX 12.4350 \\
\hline $\mathrm{Fe} x \mathrm{x}$ & 12.5260 & 7.0 & $4.46 \mathrm{e}-14$ & 5.6 & 0.16 & Fe XXI 12.4990, 12.5698, Fe XX 12.5760, 12.5760 \\
\hline Ni XIX & 12.6560 & 6.9 & $2.36 \mathrm{e}-14$ & 4.1 & 0.00 & Fe XXI 12.6490 \\
\hline Fe XXII & 12.7540 & 7.1 & $2.34 \mathrm{e}-14$ & 4.1 & 0.24 & Fe XVII 12.6950 \\
\hline $\mathrm{Fe} \mathrm{xx}$ & 12.8240 & 7.0 & $1.66 \mathrm{e}-13$ & 10.9 & 0.03 & Fe XXI 12.8220, Fe XX $12.8460,12.8640$ \\
\hline $\mathrm{Fe} \mathrm{XX}$ & 12.9650 & 7.0 & $8.63 \mathrm{e}-14$ & 7.9 & 0.12 & Fe XX $12.9120,12.9920, \mathrm{Ni}$ XX 12.9270, Fe XIX 12.9330, Fe XXII 12.9530 \\
\hline $\mathrm{Fexx}$ & 13.0610 & 7.0 & $4.47 \mathrm{e}-14$ & 5.7 & 0.06 & Fe XIX 13.0220, Fe XX 13.0240, 13.1000, Fe XXI 13.0444, 13.0831 \\
\hline $\mathrm{Fe} \mathrm{XX}$ & 13.2740 & 7.0 & $3.13 \mathrm{e}-14$ & 4.9 & -0.01 & Fe XXII 13.2360, Fe XXI 13.2487, Ni XX 13.2560, Fe XIX 13.2658, Fe XX 13.2909 \\
\hline $\mathrm{Fexx}$ & 13.3850 & 7.0 & $3.90 \mathrm{e}-14$ & 5.5 & -0.01 & Fe XX 13.3089, 13.3470, Ni XX 13.3090, Fe XVIII 13.3230, 13.3550, 13.3948 \\
\hline Ne IX & 13.4473 & 6.6 & $2.61 \mathrm{e}-13$ & 14.2 & 0.05 & Fe XIX 13.4620, 13.4970, 13.5180, Fe XXI 13.5070 \\
\hline Fe XIX & 13.6450 & 6.9 & $2.31 \mathrm{e}-14$ & 4.3 & 0.24 & Fe XX 13.6124, 13.6150, Fe XIX 13.6481 \\
\hline Ne IX & 13.6990 & 6.6 & $5.98 \mathrm{e}-14$ & 6.9 & 0.18 & Fe XIX 13.6742, 13.6752, 13.6828 \\
\hline Fe XIX & 13.7950 & 6.9 & $7.46 \mathrm{e}-14$ & 7.7 & -0.06 & Fe XIX 13.7315, 13.7458, Fe XX 13.7670, Ni XIX 13.7790 \\
\hline Fe XVII & 13.8250 & 6.8 & $3.24 \mathrm{e}-14$ & 5.1 & 0.05 & Fe XIX 13.8390, Fe XX 13.8430, Fe XVII 13.8920 \\
\hline Fe XXI & 14.0080 & 7.0 & $7.22 \mathrm{e}-14$ & 7.6 & -0.17 & Ni XIX 14.0430, 14.0770 \\
\hline Fe XVIII & 14.2080 & 6.9 & $1.06 \mathrm{e}-13$ & 9.3 & -0.13 & Fe XVIII 14.2560 , Fe XX 14.2670 \\
\hline Fe XVIII & 14.3730 & 6.9 & $8.38 \mathrm{e}-14$ & 8.4 & 0.06 & Fe XX 14.3318, 14.4207, 14.4600 Fe XVIII 14.3430, 14.4250, 14.4392 \\
\hline Fe XVIII & 14.5340 & 6.9 & $5.83 \mathrm{e}-14$ & 7.0 & 0.14 & Fe XVIII $14.4856,14.5056,14.5710,14.6011$ \\
\hline Fe XIX & 14.6640 & 6.9 & $2.94 \mathrm{e}-14$ & 5.0 & 0.22 & Fe XVIII 14.6160, 14.6884 \\
\hline Fe XIX & 14.7250 & 6.9 & $1.34 \mathrm{e}-14$ & 3.4 & -0.24 & Fe XVIII $14.7260,14.7820$, Fe XX 14.7540 \\
\hline O VIII & 14.8205 & 6.5 & $6.04 \mathrm{e}-14$ & 7.3 & 0.34 & O VIII 14.8207, Fe XX 14.8276, 14.8526, 14.8651, 14.8785, 14.9196, Fe XIX 14.9170, Fe XVIII 14.9241 \\
\hline Fe XVII & 15.0140 & 6.7 & $2.04 \mathrm{e}-13$ & 13.5 & -0.01 & Fe XIX 15.0790 \\
\hline Fe XVII & 15.2610 & 6.7 & $5.70 \mathrm{e}-14$ & 7.2 & -0.16 & O VIII $15.1760,15.1765$, Fe XIX 15.1980 \\
\hline Fe XVII & 15.4530 & 6.7 & $1.93 \mathrm{e}-14$ & 4.2 & 0.19 & Fe XIX 15.4136, Fe XVIII 15.4940, 15.5199, Fe XX 15.5170 \\
\hline Fe XVIII & 15.6250 & 6.8 & $1.67 \mathrm{e}-14$ & 3.9 & -0.15 & \\
\hline Fe XVIII & 15.8240 & 6.8 & $3.78 \mathrm{e}-14$ & 6.0 & 0.24 & Fe XVIII 15.8700 \\
\hline Fe XVIII & 16.0710 & 6.8 & $1.50 \mathrm{e}-13$ & 12.0 & 0.06 & Fe XVIII 16.0040, O VIII 16.0055, 16.0067, Fe XIX 16.1100 \\
\hline Fe XVIII & 16.1590 & 6.8 & $1.66 \mathrm{e}-14$ & 4.1 & -0.08 & Fe XVII 16.2285, Fe XIX 16.2830 \\
\hline Fe XVII & 16.7800 & 6.7 & $6.92 \mathrm{e}-14$ & 8.3 & -0.03 & \\
\hline Fe XVII & 17.0510 & 6.7 & $1.82 \mathrm{e}-13$ & 12.5 & 0.11 & Fe XVII 17.0960 \\
\hline O VIII & 18.9671 & 6.5 & $2.13 \mathrm{e}-13$ & 14.6 & -0.09 & O VIII 18.9725 \\
\hline S XIV & 24.2850 & 6.5 & $1.68 \mathrm{e}-14$ & 3.7 & 0.40 & S XIV $24.2000,24.2890$ \\
\hline N VII & 24.7792 & 6.3 & $5.34 \mathrm{e}-14$ & 6.6 & 0.00 & N VII 24.7846 \\
\hline C VI & 28.4652 & 6.2 & $2.11 \mathrm{e}-14$ & 4.3 & 0.00 & C VI 28.4663 \\
\hline S XIV & 30.4270 & 6.5 & $1.66 \mathrm{e}-14$ & 3.5 & -0.32 & S XIV 30.4690 \\
\hline No id. & 36.3980 & & $4.30 \mathrm{e}-14$ & 6.0 & $\ldots$ & (Ne X 12.132, 3rd order) \\
\hline Si XII & 44.1650 & 6.3 & $9.40 \mathrm{e}-15$ & 4.5 & -0.12 & Si XII $44.0190,44.1780$ \\
\hline Fe XVII & 50.6861 & 6.8 & $3.99 \mathrm{e}-15$ & 3.7 & 0.32 & Fe XVI 50.5550, Fe XVII 50.8544 \\
\hline No id. & 56.9000 & & $1.07 \mathrm{e}-14$ & 4.1 & $\cdots$ & (O viII 18.97, 3rd order) \\
\hline Fe XVIII & 93.9230 & 6.8 & $2.87 \mathrm{e}-14$ & 8.6 & -0.29 & Fe Xx 93.7800 \\
\hline Fe XIX & 101.5500 & 6.9 & $1.30 \mathrm{e}-14$ & 3.7 & -0.21 & \\
\hline Fe XXI & 102.2200 & 7.0 & $1.01 \mathrm{e}-14$ & 3.3 & -0.43 & \\
\hline Fe XIX & 108.3700 & 6.9 & $3.99 \mathrm{e}-14$ & 6.4 & -0.15 & \\
\hline Fe XXII & 117.1700 & 7.1 & $1.92 \mathrm{e}-14$ & 4.4 & -0.54 & \\
\hline FeXXI & 128.7300 & 7.0 & $4.25 \mathrm{e}-14$ & 4.9 & -0.31 & \\
\hline $\mathrm{Fe} X \mathrm{x}$ & 132.8500 & 7.0 & $7.64 \mathrm{e}-14$ & 6.6 & -0.38 & Fe XXIII 132.8500 \\
\hline Fe XXII & 135.7800 & 7.1 & $5.89 \mathrm{e}-14$ & 5.8 & 0.14 & \\
\hline
\end{tabular}

${ }^{a}$ Line fluxes (in erg cm $\mathrm{cm}^{-2} \mathrm{~s}^{-1}$ ) measured in Chandra/LETG AY Cet summed spectra, and corrected by the ISM absorption. log $T_{\max }$ indicates the maximum temperature $(\mathrm{K})$ of formation of the line (unweighted by the EMD). "Ratio" is the $\log \left(F_{\mathrm{obs}} / F_{\text {pred }}\right)$ of the line. Blends amounting to more than $5 \%$ of the total flux for each line are indicated. For some lines not identified in APED, a tentative identification as 3rd order emission of intense lines is suggested in the "Blends" column. 